\title{
Composed endotypes to guide antibiotic discontinuation in sepsis
}

\author{
Jesus F. Bermejo-Martin ${ }^{1,2^{*}}$ (D, David Andaluz-Ojeda ${ }^{1,3}$, Marta Martin-Fernandez ${ }^{1}$, Cesar Aldecoa ${ }^{1,4}$ and \\ Raquel Almansa ${ }^{1,2}$
}

\begin{abstract}
Overuse of empiric antibiotic therapy in the ICU is responsible for promoting the dissemination of multidrugresistant (MDR) bacteria. Shortened antibiotic treatment duration could contribute to palliating the emergence of MDR. Uncertainty about patient evolution is a major concern for deciding to stop antibiotics. Biomarkers could represent a complementary tool to identify those patients for whom antibiotic treatment could be safely discontinued. The biomarker most extensively studied to guide antibiotic withdrawal is procalcitonin (PCT), but its real impact on decreasing the duration of antibiotic treatment is a matter of controversy. Combining biomarkers to rule out complicated outcomes in sepsis patients could represent a better option. Some candidate biomarkers, including mid-regional proadrenomedullin, the percentage of human leukocyte antigen DR (HLA-DR)-positive monocytes, means of fluorescence intensities of HLA-DR on monocytes, interleukin-7 receptor expression levels, immunoglobulin $\mathrm{M}$ levels in the serum or the absence of increased proteolysis, have already demonstrated the potential to exclude the risk of progression to septic shock, nosocomial infections, and mortality when tested along the sepsis course. Other promising biomarkers to rule out complicated outcomes are neutrophil protease activity, the adaptive/coagulopathic signatures identified by whole transcriptome analysis by Sweeney et al., and the SRS1 signature identified by Davenport et al. In conclusion, there are a number of promising biomarkers involved in proteolytic, vascular, immunological, and coagulation alterations that could be useful to build composed endotypes to predict uncomplicated outcomes in sepsis. These endotypes could help to identify patients deserving the discontinuation of antibiotics.
\end{abstract}

Keywords: Empiric, Antibiotic, Treatment, Sepsis, Severity, Endotypes

Overuse of empiric antibiotic therapy in the ICU is responsible for promoting the dissemination of multidrug-resistant (MDR) bacteria [1]. De-escalation and shortened antibiotic treatment duration are strategies that could contribute to palliating the emergence of MDR [1]. Antibiotic stewardship programs are proposed considering antibiotic cessation in patients exhibiting clinical improvement, but uncertainty about patient evolution is a major concern for deciding to stop antibiotics. Biomarkers could represent a complementary

\footnotetext{
* Correspondence: jfbermejo@saludcastillayleon.es

'Group for Biomedical Research in Sepsis (BioSepsis), Hospital Clínico Universitario de Valladolid/IECSCYL, Av. Ramón y Cajal, 3, 47003 Valladolid, Spain

${ }^{2}$ Centro de Investigación Biomedica en Red-Enfermedades Respiratorias (CibeRes, CB06/06/0028), Instituto de salud Carlos III (ISCIII), Av. de Monforte de Lemos, 5, 28029 Madrid, Spain

Full list of author information is available at the end of the article
}

tool to identify patients for whom antibiotic treatment could be safely discontinued. The biomarker most extensively studied to guide antibiotic withdrawal is procalcitonin (PCT). A recent meta-analysis by Lam et al. showed that PCT guidance could help to decrease antibiotic duration and even mortality [2]. In contrast, Pepper et al. concluded that the increased survival and decreased antibiotic utilization associated with PCTguided antibiotic discontinuation represent low certainty evidence [3].

Relying on a single biomarker is probably not the most appropriate strategy to identify patients deserving antibiotic discontinuation. There are a number of biomarkers that inform the evolution of organ failure and prognosis during sepsis. These biomarkers could be used to build composed endotypes to rule out complicated

(c) The Author(s). 2019 Open Access This article is distributed under the terms of the Creative Commons Attribution 4.0 International License (http://creativecommons.org/licenses/by/4.0/), which permits unrestricted use, distribution, and reproduction in any medium, provided you give appropriate credit to the original author(s) and the source, provide a link to the Creative Commons license, and indicate if changes were made. The Creative Commons Public Domain Dedication waiver (http://creativecommons.org/publicdomain/zero/1.0/) applies to the data made available in this article, unless otherwise stated. 
outcomes, helping to identify the patients qualifying for antibiotic cessation.

\section{Biomarkers of microcirculatory damage}

Adrenomedullin is a peptide produced by multiple tissues during physiological and infectious stress with varying functions (Fig. 1). Levels of the stable mid-regional fragment of proadrenomedullin (MR-proADM) correlate with organ failure degree in sepsis. Elke et al. showed that low MR-proADM concentrations identify sepsis patients with a very low risk of death throughout the ICU stay [4]. A value of MR-ProADM $<2.25 \mathrm{nmol} / \mathrm{L}$ at day 4 or day 7 following ICU admission yielded a negative predictive value (NPV) of $94 \%$ to rule out 28 -day mortality. In turn, this cutoff yielded an NPV of $96 \%$ at day 10 [4].

\section{Immunological biomarkers}

Human leukocyte antigen DR (HLA-DR) represents the capacity of monocytes for antigen presentation and crosstalk to $T$ helper cells, enabling the activation of the adaptive immune system. HLA-DR on monocytes is considered a surrogate marker of sepsis-induced immunosuppression. Survivors show a significant increase in the percentage of HLA-DR-positive monocytes during the course of sepsis [5]. Using flow cytometry in a cohort of patients with severe sepsis, Wu et al. demonstrated that an increment of $4.8 \%$ at day 3 following admission to the ICU yielded a NPV of $98 \%$ to rule out mortality at 28 days, while an increment of $9 \%$ at day 7 yielded an NPV of $97.2 \%$ [6]. Landelle et al. also employed flow cytometry and demonstrated that monocyte HLA-DR expressed as the means of fluorescence intensity (MFI) is useful to rule out nosocomial infections after septic shock. Exhibiting > 54 MFIs by days 3-4 following admission to the ICU showed an NPV of $86 \%$ to exclude nosocomial infections in these patients [7]. Another promising biological marker to rule out complicated outcomes in sepsis patients during hospitalization in the ICU is interleukin-7 receptor (IL-7R). Interleukin 7 is primarily a survival factor for different subpopulations of lymphoid cells. Using reverse transcription quantitative polymerase chain reaction in whole blood, Delwarde et al. found a gene expression value for IL-7R at day 3 following septic shock diagnosis that yielded a high NPV for 28 -day mortality (86\%) [8]. This value corresponded to a ratio of 0.20 between the expression of IL-7R and hypoxanthine phosphoribosyltransferase 1 as a reference gene. Immunoglobulin $M$ levels in serum could also help to identify patients with better outcomes. The work of Giamarellos-Bourboulis EJ et al. supported that the kinetics of immunoglobulin $\mathrm{M}$ levels in sepsis patients over time could help to rule out progression to septic shock and mortality [9]. Using advanced informatic techniques, Sweeney et al. evidenced the existence

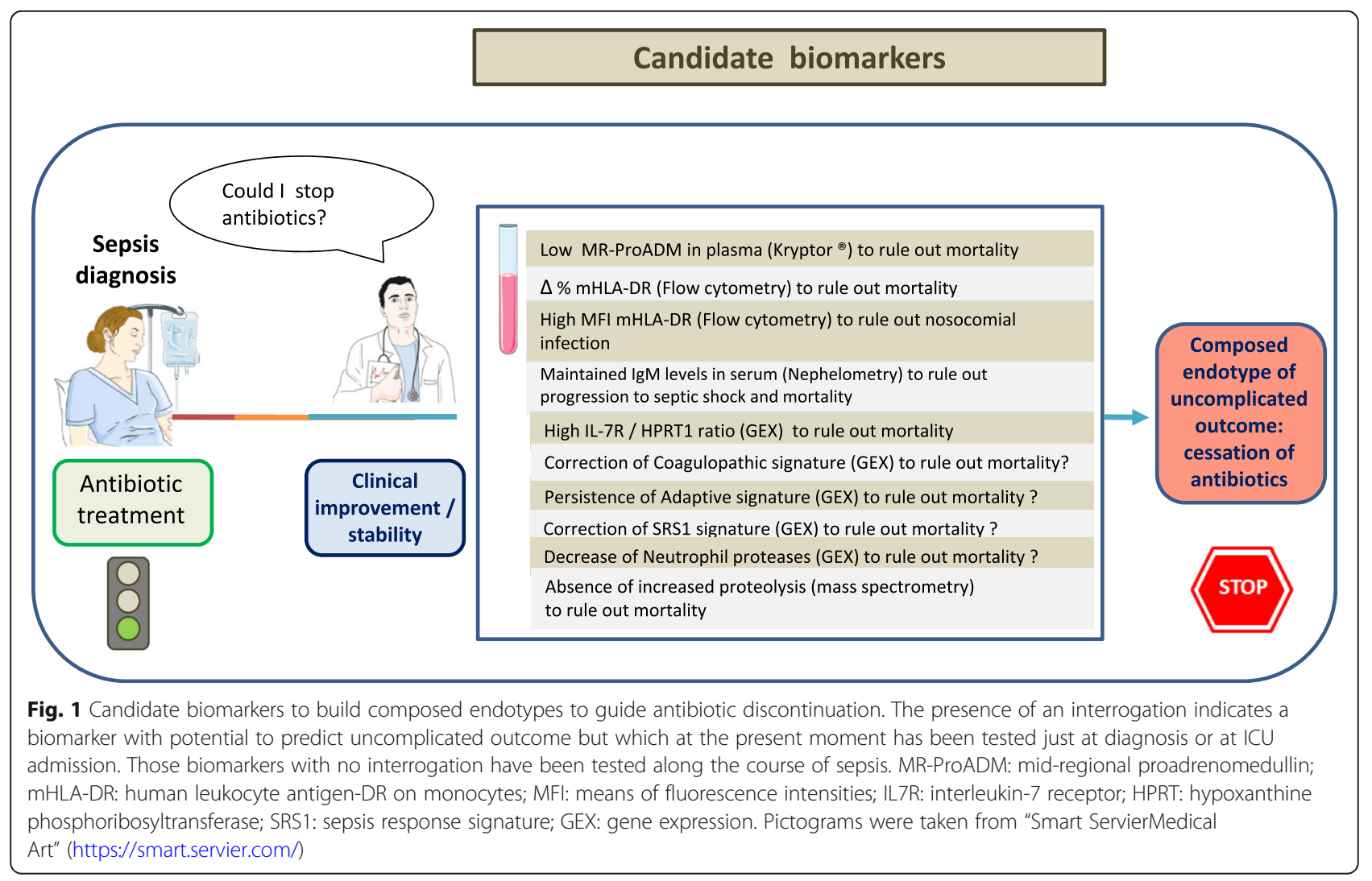


in sepsis of a gene expression signature of adaptive immune activation associated with a lower clinical severity and lower mortality [10]. Similarly, Davenport et al. identified a sepsis response signature 1 (SRS1) characterizing individuals with an immunosuppressed phenotype that included features of endotoxin tolerance, $\mathrm{T}$ cell exhaustion, and the downregulation of human leucocyte antigen class II, which was associated with higher 14-day mortality [11]. The persistence/presence of the "adaptive" signature or the correction/absence of the SRS1 signature during sepsis could potentially help to identify patients at low risk of developing complications.

\section{Coagulation and neutrophil protease related biomarkers}

Sweeney et al. also found the existence of a gene expression signature of clinical coagulopathy that is associated with higher mortality in sepsis [10]. Evidence of the correction/absence of this signature could also help to identify low-risk patients. Neutrophil proteases are involved in the pathogenesis of the coagulation alterations observed in sepsis and mediate endothelial damage. We have identified that neutrophil protease activity (matrix metallopeptidase 8-MMP8 and lipocalin-2-LCN2/NGAL) is associated with organ failure degree and mortality in sepsis [12, 13]. Evidence of low/decreased levels of neutrophil proteases during hospitalization is also an additional candidate signal to identify uncomplicated patients. Neutrophil proteases mediate proteolysis [14]. Using mass spectrometry, Bauzá-Martinez et al. demonstrated that septic shock survivors are characterized by the absence of increased proteolysis along the evolution of the disease (evidenced by peptide abundance) [14].

\section{Conclusion}

There are a number of promising biomarkers involved in proteolytic, vascular, immunological, and coagulation alterations that could be useful to build composed endotypes predicting uncomplicated outcomes in sepsis. These endotypes could help to identify patients deserving of the discontinuation of antibiotics. Further efforts are needed to identify the optimal biomarker combinations to obtain the best-composed endotype/s identifying patients with uncomplicated outcomes by single or repeated evaluations along the course of the disease. New biomarker profiling technologies, such as automated bedside flow cytometers (Accellix) [15], microfluidic immunoassays (Simple-Plex), and next generation mRNA quantification technologies (droplet digital PCR [13], Nanostring), will facilitate the accurate identification of these composed endotypes.

\section{Abbreviations}

HLA-DR: Human leukocyte antigen-DR; HPRT1: Hypoxanthine

phosphoribosyltransferase 1 as reference gene; IL7R: Interleukin-7 Receptor messenger RNA; MFI: Means of fluorescence intensities; MR-ProADM: Midregional proadrenomedullin; SRS1: Sepsis response signature 1

\section{Acknowledgements \\ We thank the Anesthesiology and Critical Care Services of our Hospitals for their continued support to our research program on sepsis.}

\section{Funding}

We thank the "Instituto de Salud Carlos III" and "Consejeria de Sanidad de Castilla y Leon" for their financial support, grant numbers [PI15/01959][PI16/ 01156] and "Consejería de Educación de Castilla y Leon/Fondo social Europeo" for supporting the contract of Marta Martin-Fernandez. This research group is supported with funds from "Fondo Europeo de Desarrollo Regional, Una manera de hacer Europa."

\section{Availability of data and materials} Not applicable

\section{Authors' contributions}

JFBM wrote the manuscript. MMF and RA created the figure. JFBM, DAO, MMF, CA, and RA critically reviewed the manuscript and provided their suggestions to improve it. All authors read and approved the final manuscript.

\section{Authors' information}

BioSepsis is a translational research group working at the National Health System-SACYL in Spain devoted to better understand the pathogenesis of sepsis and to identify new biomarkers and treatments to improve the care of sepsis patients.

\section{Ethics approval and consent to participate}

Not applicable

\section{Consent for publication}

Not applicable

\section{Competing interests}

The authors declare that they have no competing interests.

\section{Publisher's Note}

Springer Nature remains neutral with regard to jurisdictional claims in published maps and institutional affiliations.

\section{Author details}

${ }_{1}^{1}$ Group for Biomedical Research in Sepsis (BioSepsis), Hospital Clínico Universitario de Valladolid/IECSCYL, Av. Ramón y Cajal, 3, 47003 Valladolid, Spain. ${ }^{2}$ Centro de Investigación Biomedica en Red-Enfermedades Respiratorias (CibeRes, CB06/06/0028), Instituto de salud Carlos III (ISCIII), Av. de Monforte de Lemos, 5, 28029 Madrid, Spain. Intensive Care Medicine Service, Hospital Clínico Universitario de Valladolid/IECSCYL, Av. Ramón y Cajal, 3, 47003 Valladolid, Spain. ${ }^{4}$ Anesthesiology and Reanimation Service, Hospital Universitario Río Hortega, Calle Dulzaina, 2, 47012 Valladolid, Spain.

Received: 15 December 2018 Accepted: 15 April 2019

Published online: 24 April 2019

\section{References}

1. Timsit J-F, Bassetti M, Cremer O, Daikos G, de Waele J, Kallil A, et al. Rationalizing antimicrobial therapy in the ICU: a narrative review. Intensive Care Med. 2019;45:172-89.

2. Lam SW, Bauer SR, Fowler R, Duggal A. Systematic review and meta-analysis of procalcitonin-guidance versus usual care for antimicrobial management in critically ill patients: focus on subgroups based on antibiotic initiation, cessation, or mixed strategies. Crit Care Med. 2018;46:684-90.

3. Pepper D, Sun J, Rhee C, Welsh J, Powers JH, Danner RL, et al. Procalcitoninguided antibiotic discontinuation and mortality in critically ill adults: a systematic review and meta-analysis. Chest. 2019.

4. Elke G, Bloos F, Wilson DC, Brunkhorst FM, Briegel J, Reinhart K, et al. The use of mid-regional proadrenomedullin to identify disease severity and treatment response to sepsis - a secondary analysis of a large randomised controlled trial. Crit Care. 2018;22:79. 
5. Monneret G, Lepape A, Voirin N, Bohé J, Venet F, Debard A-L, et al. Persisting low monocyte human leukocyte antigen-DR expression predicts mortality in septic shock. Intensive Care Med. 2006;32:1175-83.

6. Wu J-F, Ma J, Chen J, Ou-Yang B, Chen M-Y, Li L-F, et al. Changes of monocyte human leukocyte antigen-DR expression as a reliable predictor of mortality in severe sepsis. Crit Care. 2011;15:R220.

7. Landelle C, Lepape A, Voirin N, Tognet E, Venet F, Bohé J, et al. Low monocyte human leukocyte antigen-DR is independently associated with nosocomial infections after septic shock. Intensive Care Med. 2010;36:1859-66.

8. Delwarde B, Peronnet E, Venet F, Cerrato E, Meunier B, Mouillaux J, et al. Low interleukin-7 receptor messenger RNA expression is independently associated with day 28 mortality in septic shock patients. Crit Care Med. 2018;46:1739-46.

9. Giamarellos-Bourboulis EJ, Apostolidou E, Lada M, Perdios I, Gatselis NK, Tsangaris I, et al. Kinetics of circulating immunoglobulin M in sepsis: relationship with final outcome. Crit Care. 2013;17:R247.

10. Sweeney TE, Azad TD, Donato M, Haynes WA, Perumal TM, Henao R, et al. Unsupervised analysis of transcriptomics in bacterial Sepsis across multiple datasets reveals three robust clusters. Crit Care Med. 2018;46:915-25.

11. Davenport EE, Burnham KL, Radhakrishnan J, Humburg P, Hutton P, Mills TC, et al. Genomic landscape of the individual host response and outcomes in sepsis: a prospective cohort study. Lancet Respir Med. 2016:4:259-71.

12. Almansa R, Heredia-Rodríguez M, Gomez-Sanchez E, Andaluz-Ojeda D, Iglesias $\mathrm{V}$, Rico L, et al. Transcriptomic correlates of organ failure extent in sepsis. J Inf Secur. 2015;70:445-56.

13. Almansa R, Ortega A, Ávila-Alonso A, Heredia-Rodríquez M, Martín S, Benavides $D$, et al. Quantification of immune dysregulation by nextgeneration polymerase chain reaction to improve Sepsis diagnosis in surgical patients. Ann Surg. 2019;269:545-53.

14. Bauzá-Martinez J, Aletti F, Pinto BB, Ribas V, Odena MA, Díaz R, et al. Proteolysis in septic shock patients: plasma peptidomic patterns are associated with mortality. Br J Anaesth. 2018;121:1065-74.

15. Zouiouich M, Gossez M, Venet F, Rimmelé T, Monneret G. Automated bedside flow cytometer for mHLA-DR expression measurement: a comparison study with reference protocol. Intensive Care Med Exp. 2017:5:39.

Ready to submit your research? Choose BMC and benefit from:

- fast, convenient online submission

- thorough peer review by experienced researchers in your field

- rapid publication on acceptance

- support for research data, including large and complex data types

- gold Open Access which fosters wider collaboration and increased citations

- maximum visibility for your research: over $100 \mathrm{M}$ website views per year

At $\mathrm{BMC}$, research is always in progress.

Learn more biomedcentral.com/submissions 